\title{
Comparative Evaluation of Manual and Automated Irrigation Systems in the Arthroscopic Repair of Rotator Cuff Tears
}

\author{
Assoc. Prof. Olcay Güler, MD $^{1 *}$, Gürkan Gümüşsuyu, MD $^{2}$ \\ ${ }^{I}$ Department of Orthopedics and Traumatology, Altinbas University, Medical Faculty, Istanbul, Turkey \\ ${ }^{2}$ Department of Orthopedics and Traumatology, Istinye University, Medical Faculty, Istanbul, Turkey
}

*Corresponding Author: Assoc. Prof. Olcay Guler, MD, Department of Orthopedics and Traumatology, Altınbaş University Medical Faculty, Bağcılar, Istanbul, Turkey. Email: olcayguler77@gmail.com

\begin{abstract}
Purpose: Rotator cuff tears (RCT) can be treated arthroscopically, and intra-articular irrigation is an important method that facilitates the surgical procedure. We aimed to comparatively evaluate the clinical outcomes in arthroscopic repair of RCTs operated using either manual or automated pressure adjusted intraarticular irrigation systems.

Methods: Retrospective analysis of data collected from the medical files of 69 patients (62 males, 7 males) who underwent arthroscopy for RCT. Patients were allocated into two groups as for the type of intraarticular irrigation method: Group I $(n=38)$ received automated intra-articular irrigation, whereas Group II $(n=31)$ had manual irrigation during surgery. Demographic information, clinical and perioperative data, and therapeutic outcomes were compared between two groups.

Results: The average age of our population was $60.61 \pm 6.56$ (range, 42 to 72) years. There was no statistically significant difference between two groups in terms of age, sex distribution, body-mass index, comorbidities, muscle atrophy as per Goutallier classification, size and shape of RCTs, the amount of isotonic saline used for irrigation, operative time, length of in-hospital stay, preoperative and postoperative ConstantMurley scores, additional surgical procedures, complication rates and duration of follow-up.

Conclusion: Good visualization is mandatory during arthroscopic surgery for RCTs. The maintenance of a clear optical medium necessitates an effective irrigation system. Our data imply that manual irrigation systems can offer a safe, effective, and practical alternative that provides effective vision during arthroscopy for RCTs.
\end{abstract}

Keywords: rotator cuff tear; arthroscopy; intra-articular irrigation; manual; automated.

\section{INTRODUCTION}

Rotator cuff tears (RCT) are common pathologies which affect middle-aged and elderly population with a prevalence ranging between $4 \%$ to $54 \%$ (1). Symptomatic RCTs can be managed with conservative and surgical treatment modalities (2). There is still controversy on the indications of surgical treatment of RCTs. Still, parameters such as duration and severity of symptoms, shape, and size of the tear, muscle atrophy, and fatty infiltration may influence the selection of the mode of treatment $(2,3)$. Despite the shortage of evidence-based agreement for indications, surgery has been performed as the mainstay of treatment and the technique has insidiously shifted from the open approach to the mini-open and subsequently to arthroscopic techniques (4, $5)$.
Recently, arthroscopic techniques were popularized attributed to its advantages such as smaller skin incisions, access to the glenohumeral joint and less soft tissue dissection (6). Some studies suggest that arthroscopy can be administered for not only small and medium-sized RCTs, but also larger tears $(>3 \mathrm{~cm})$ can be treated successfully (7).

On the other hand, surgical procedures using the arthroscopic technique can be challenging and they may necessitate practice and guidance. The procedure can be enhanced by modern arthroscopic telescopes and special instruments as well as forceful irrigation of the joint which allows visualization in the presence of blood or blurred joint fluid (8).

Bergstrom et al. suggested the utility of an infusion pump for providing a more effective 
distention of the joint. In this manner, higher intraarticular pressures and greater rates of flow can be achieved, and the flow can be preserved in the presence of greater pressure. The utility of gravity systems alone may lead to cessation of flow when distention is obtained. On the other hand, increasing the flow rate may aid in the cleansing of blood and debris (8).

Visualization is a critical point for the implementation of safe and effective arthroscopic procedures. Recently, remarkable improvements have been attained by using pump systems to increase fluid flow. This allows distension of the joint and cleansing the joint of blood and debris and this method gained more importance with the use of power equipment such as the suction shavers that tend to remove fluid from the joint rapidly and hinder sufficient visualization (9).

The relationship between different irrigation systems and clinical outcomes during arthroscopy for RCTs has not been yet elucidated. We aimed to compare the clinical and functional outcomes of rotator cuff tears who underwent arthroscopic repair for RCTs with either manual or automated pressureadjusted irrigation systems.

\section{MATERIALS AND METHODS}

\subsection{Study Design}

This retrospective study was performed in the orthopaedics and traumatology department of a university hospital. Written informed consent was obtained from all subjects. The procedures were performed in accordance with the principles of the Helsinki Declaration.

Data were extracted from the medical files of 69 patients (62 females, 7 males) with an average age of $60.61 \pm 6.56$ (range: 42 to 72 ). These patients underwent arthroscopy between January 2016 and January 2018 for small or medium-sized rotator cuff tears which were unresponsive to conservative treatment.

The inclusion criteria were small to mediumsized tears on magnetic resonance imaging (MRI) and availability for a minimum of 2 years of follow-up after surgery. The exclusion criteria were large and massive rotator cuff tears, subscapular tears, arthrosis, history of trauma or previous surgery, patients lost to follow-up, and collagen vascular diseases.

All patients were operated by the same surgeon at the beach chair position under hypotensive anesthesia. After the evaluation of the glenohumeral joint, subacromial space was passed. Acromioplasty was routinely performed in all cases, while biceps tenotomy was selectively carried out in 37 patients. The affected arm was maintained in a sling for 6 weeks. Pendular movements were started on the $1^{\text {st }}$ postoperative day and physical medicine \& rehabilitation were recommended thereafter. A pressure-controlled-pump (Smith \& Nephew DYONICS 25 Fluid Management System ${ }^{\circledR}$, Smith \& Nephew Dyonics, Andover, MA, USA) was used in Group I $(n=38)$ during arthroscopy. The pump device was set for an isotonic saline volume of $3,000 \mathrm{~mL}(0.9 \%$ isotonic sodium chloride, Polifleks ${ }^{\circledR}, \quad$ Polifarma, Istanbul, Turkey) with a pressure that allows clear visualization of the surgical field.

On the other hand, a manual irrigation pump (Bıçakçılar ${ }^{\circledR}$, Şişli, Istanbul, Turkey) was used in Group II $(\mathrm{n}=31)$. A total of $3,000 \mathrm{~mL}$ isotonic saline was introduced at a level $150 \mathrm{~cm}$ above the ground. Demographic data, clinical outcomes, duration of surgery, complications, and cost of irrigation systems were compared between two groups. Muscle atrophy was assessed per Goutallier classification which had five stages, ranging from Stage 0 (normal muscle) to Stage 4 (more fat than muscle) and the fatty infiltration was characterized by areas of decreased radiodensity (10). Rotator cuff tears were categorized as either small $(<1 \mathrm{~cm})$, medium $(1-3 \mathrm{~cm})$, large $(3-5 \mathrm{~cm})$, or massive $(>5 \mathrm{~cm})(11)$. The tear pattern was classified at the time of surgery as crescent, U-shaped, or Lshaped as reported in the relevant literature (12).

The Constant-Murley Score (CMS) was developed for the assessment of overall shoulder function, irrespective of diagnosis and it has been approved and has been widely used since then $(13,14)$.

Descriptive data, clinical features, therapeutic outcomes, duration of surgery, and complications were compared between two groups. The number of anchors, comorbidities, duration of procedures at the subacromial level and glenohumeral joints, amount of isotonic saline used for intra-articular irrigation, CMS preoperatively and at the final control, duration of in-hospital stay and complications were investigated.

\subsection{Statistical Analysis}

All of our data were analyzed with Statistical Package for Social Sciences program version 
20.0 (SPSS Inc., Chicago, IL, USA). Descriptive statistical analysis was performed for age and sex variables. Before the comparison of two groups, normality was tested with the ShapiroWilk test for quantitative continuous variables. The level of significance $>0.05$ was assumed as consistent with the normal distribution. Variables with normal distribution were compared using the T-test, while the MannWhitney $U$ test was employed for variables without normal distribution. A Chi-square test was used for the evaluation of categorical variables. A p-value $<0.05$ was considered as statistically significant.

\section{Results}

Data were extracted from the medical files of 69 patients (62 females, 7 males) with an average age of $60.61 \pm 6.56$ (range, 42 to 72 ) years. Group I $(n=38)$ consisted of 34 women and 4 men with an average age of $60.55 \pm 6.36$ years, while Group II $(n=31)$ was comprised of 31 patients ( 28 women, 3 men) with an average age of $60.68 \pm 6.91$ years.

An overview of demographic and clinical data is presented in Table 1. There were no remarkable differences between two groups as per age $(\mathrm{p}=0.938)$, sex distribution $(\mathrm{p}=0.908)$, side of involvement $\quad(\mathrm{p}=0.815)$, body-mass index $(\mathrm{p}=0.406)$, and comorbidities $(\mathrm{p}=0.823)$. Thus, baseline descriptives displayed similar features in both groups. Two groups were also similar the severity of muscle atrophy ( $\mathrm{p}=0.812)$.

Table 1. A comparative overview of demographic and clinical variables in 2 groups

\begin{tabular}{|c|c|c|c|c|}
\hline \multirow{2}{*}{\multicolumn{2}{|c|}{ Variable }} & \multicolumn{2}{|c|}{ Groups } & \multirow{2}{*}{ Value } \\
\hline & & $\begin{array}{l}\text { Group } \\
\text { I } \\
(n=38)\end{array}$ & $\begin{array}{l}\text { Group } \\
\text { II } \\
(\mathbf{n}=\mathbf{3 1})\end{array}$ & \\
\hline \multicolumn{2}{|l|}{ Age (years) } & $\begin{array}{l}60.55 \\
\pm 6.36 \\
\end{array}$ & \begin{tabular}{|l|}
60.68 \\
\pm 6.91 \\
\end{tabular} & 0.938 \\
\hline \multicolumn{2}{|l|}{ Sex distribution (F/M) } & $34 / 4$ & $28 / 3$ & 0.908 \\
\hline \multicolumn{2}{|l|}{ Side of involvement (R/L) } & $17 / 21$ & $13 / 18$ & 0.815 \\
\hline \multicolumn{2}{|l|}{ Body-mass index $\left(\mathrm{kg} / \mathrm{m}^{2}\right)$} & 34.86 & 35.18 & 0.946 \\
\hline \multirow[t]{2}{*}{ Comorbidities } & $\mathrm{DM}$ & 2 & 1 & \multirow[t]{2}{*}{0.823} \\
\hline & HT & 15 & 10 & \\
\hline \multirow{3}{*}{$\begin{array}{l}\text { Muscle atrophy as for } \\
\text { Goutallier classification }\end{array}$} & 0 & 17 & 16 & \multirow[t]{3}{*}{0.812} \\
\hline & 1 & 19 & 14 & \\
\hline & 2 & 2 & 1 & \\
\hline
\end{tabular}

(Abbreviations: F: female; M: male; R: right; L: left; DM: diabetes mellitus; HT: hypertension)

Table 2 displays a comparative presentation of operative and postoperative data in our series. The size $(\mathrm{p}=0.873)$, and shape of RCT $(\mathrm{p}=0.898)$, number of suture anchors $(\mathrm{p}=0.536)$, and sequence of $\mathrm{RC}$ repair $(\mathrm{p}=0.646)$. The average perioperative pump pressure in Group I was $57.63 \pm 4.90$ (range, 50 to 65 ) $\mathrm{mmHg}$. No differences were noted between two groups in terms of isotonic saline used for irrigation $(\mathrm{p}=0.969)$, duration of operation $(\mathrm{p}=0.790)$, length of hospital stay $(\mathrm{p}=0.922)$, preoperative and postoperative CMSs $(\mathrm{p}=0.718$ and $\mathrm{p}=0.720$, respectively), complication rates $(\mathrm{p}=0.862)$, and duration of follow-up ( $\mathrm{p}=0.791)$ (Table 2).

Table 2. An overview of operative and postoperative data collected from our series

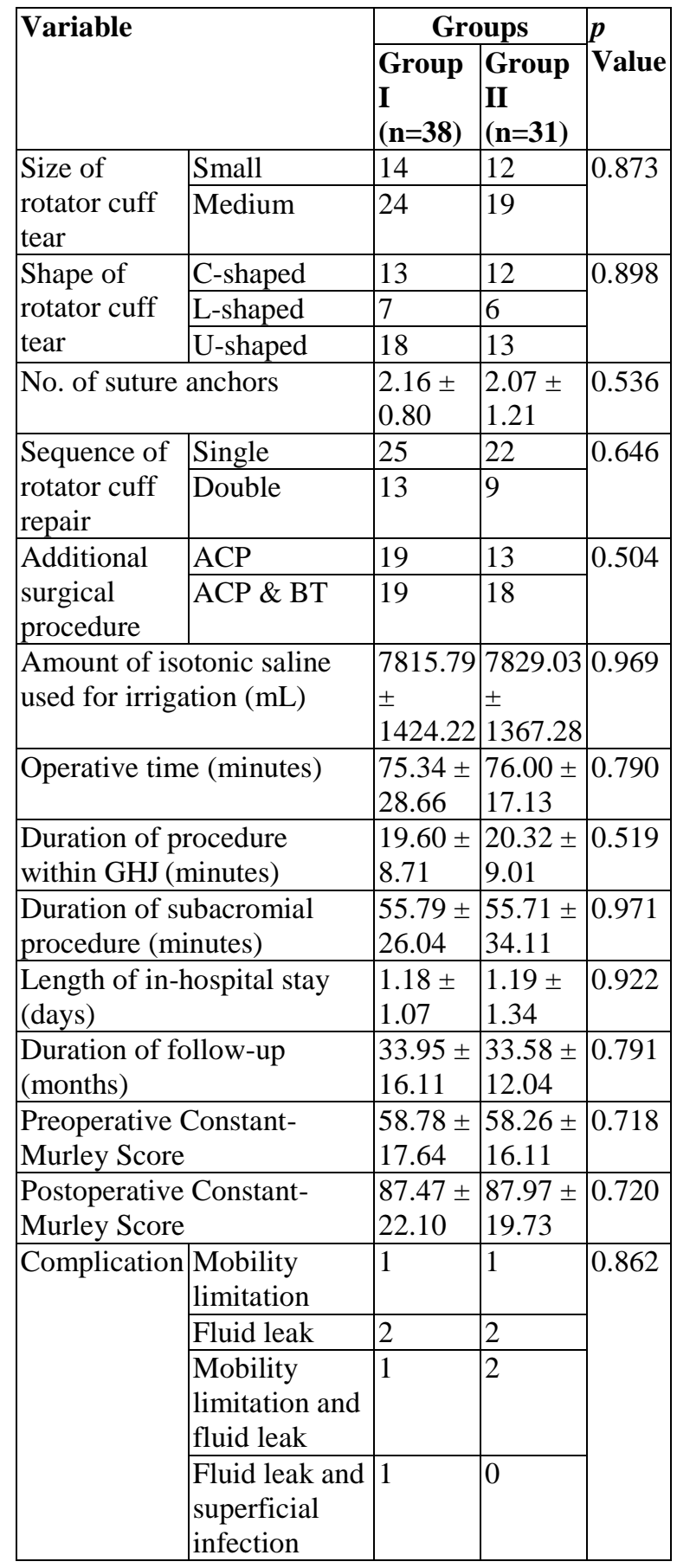

(Abbreviations: ACP: acromioplasty; BT: biceps tenotomy; GHJ: glenohumeral joint) 


\section{DisCUSSION}

The synovial fluid does not constitute an optimal medium for visualization and is replaced either by gas or fluid for arthroscopy. The surgeons mostly prefer the fluid medium, but there is variability for the mode of irrigation (15).

Pump systems used in arthroscopic surgery have recently evolved to refine the intraoperative visualization. Gravity flow systems were previously developed and are still in use. Automated pump systems with pressure or pressure and volume control have been developed. The automated irrigation systems offer some advantages over gravity irrigation such as a more consistent flow, a greater degree of joint distention, refined visualization especially with motorized instrumentation, less requirement for tourniquet use, a tamponade effect on bleeding, and decreased operative time. Disadvantages of automated irrigation systems involve the need for additional equipment with increased cost and maintenance, the initial learning curve for the surgical team, and the amplified risk of extra-articular fluid dissection and associated complications like compartment syndrome. In parallel with the improvement of quality and pump systems, the index of diagnostic and therapeutic focusing on the joint pathology will also expand $(15,16)$.

Recently, arthroscopy has been popularized to treat a wide range of shoulder pathologies. Sufficient visualization during these procedures is of utmost importance for the effective performance of the arthroscopic procedure. Hemostasis, hypotensive anesthesia, and the use of irrigation systems are the common methods to optimize adequate visualization during arthroscopy $(16,17)$. We aimed to determine whether there were any advantages or significant differences between RCT patients who underwent arthroscopy using manual or automated pressure-adjusted irrigation systems. Our results indicated that there were no significant differences between demographic and clinical features as well as therapeutic outcomes in these two groups. Thus, our data imply that manual irrigation systems can constitute a cost-effective, cheap, and safe alternative to automated pressure-adjusted irrigation systems in arthroscopy for RCTs. Cost issues of surgical procedures are supposed to gain more importance soon due to the worldwide stagnancy attributed to the COVID19 pandemic.
In conjunction with the report by Ogilvie-Harris et al., the more sophisticated automated pump did not consume larger fluid volume during the operation (9). Neither the duration of the procedure nor complications and functional outcomes displayed any remarkable differences between two groups.

The irrigation method used during arthroscopy must be technically easy and must be capable of improving the visualization of the surgical field (17). The pros and cons such as practical benefit and the additional cost associated with the selection of the irrigation system must be analyzed carefully. Both the effectivity of visualization and maintenance of patient safety are important parameters for the utility of irrigation pump systems during arthroscopy. Visualization is dependent on the camera image quality and preservation of a clear fluid medium and the irrigation system preserves the fluid medium environment. To provide patient safety, low intra-articular pressure, and a low level of fluid extravasation must be maintained. The ease of set-up and low complication rates are important criteria for the selection of irrigation systems $(15,16)$. An optimal automated pump must be able to distribute the essential flow rate, maintain the intra-articular pressure at ideal levels needed for visualization, and must possess safety properties like pressure monitors (18). Even though manual regulation of flow for adjustment of appropriate vision may be troublesome, our data yielded that there were no noteworthy differences between manual and automated pressure adjusted irrigation systems in terms of perioperative parameters and clinical outcomes. Manual irrigation systems had been used safely and without any significant complications (19).

There is no consensus regarding the best method of irrigation to use during arthroscopic surgery (19). We noted that the manual irrigation system was as effective as an automated irrigation system to achieve an adequate vision of the surgical field. The manual irrigation system can provide a safe and effective alternative in conditions without the availability of automated systems. Familiarity and experience of the surgical team with the irrigation method selected for the arthroscopic procedure is an important aspect to be remembered during preoperative planning.

Diagnostic and therapeutic arthroscopy can be enhanced if the surgeon can directly control the 
flow and pressure to rapidly achieve and maintain a clear vision of the operative field (16). We have utilized suture anchors during all arthroscopic repairs of RCTs. As stated by Aleem et al., we support that they provide a sufficient fixation strength to the bone (5). There was a predominance of females in our series $(89.8 \%)$ and this was in contrast to some relevant publications (20). This difference may be attributed to many factors such as increased involvement in women in labor, diminution of muscle strength more prominently in females, and increased awareness to seek medical care.

The main limitations of the present study involve retrospective design, small sample size, the predominance of female patients in our series, data confined to the experience of a single-center, and other confounding factors such as ethnic, socio-economical, and genetic aspects. Therefore, extrapolation of our results to larger populations must be made cautiously.

In conclusion, the results of the present study indicated there were no significant differences between manual and automated irrigation systems in terms of perioperative parameters, clinical outcomes, and complication rates. Manual irrigation systems can offer a safe, cheap, and practical alternative to automated irrigation systems $\mathrm{s}$ provided effective vision during arthroscopy for RCTs. Further prospective, controlled trials on larger series are warranted to reach more accurate conclusions.

\section{SOURCE OF FUNDING}

No financial support was received for this paper.

\section{ACKNOWLEDGMENTS}

None

\section{CONFLICT OF INTEREST}

The authors declare no competing interests

\section{REFERENCES}

[1] Bartolozzi A, Andreychik D, Ahmad S (1994) Determinants of outcome in the treatment of rotator cuff disease. Clin Orthop Relat Res 308:90-97.

[2] Wolf BR, Dunn WR, Wright RW (2007) Indications for repair of full-thickness rotator cuff tears. Am J Sports Med 35(6):1007-1016.

[3] Favard L, Bacle G, Berhouet J (2007) Rotator cuff repair. Joint Bone Spine 74(6):551-557.

[4] Dunn WR, Schackman BR, Walsh C, Lyman S, Jones EC, Warren RF, et al. (2005) Variation in orthopaedic surgeons' perceptions about the indications for rotator cuff surgery. J Bone Joint Surg Am 87(9):1978-1984.

[5] Aleem AW, Brophy RH (2012) Outcomes of rotator cuff surgery: what does the evidence tell us? Clin Sports Med. 31(4):665-674.

[6] Gartsman GM, Khan M, Hammerman SM (1998) Arthroscopic repair of full-thickness

[7] tears of the rotator cuff. J Bone Joint Surg Am 80(6):832-840.

[8] Burkhart SS, Lo IK (2006) Arthroscopic rotator cuff repair. J Am Acad Orthop Surg 14(6):333346.

[9] Bergstrom R, Gillquist J (1986) The use of an infusion pump in arthroscopy. Arthroscopy. 2(1):41-45.

[10] Ogilvie-Harris DJ, Weisleder L (1995) Fluid pump systems for arthroscopy: a comparison of pressure control versus pressure and flow control. Arthroscopy. 11(5):591-595.

[11] Goutallier D, Postel JM, Bernageau J, Lavau L, Voisin MC (1994) Fatty muscle degeneration in cuff ruptures: pre- and postoperative evaluation by CT scan. Clin Orthop Relat Res. 304:78-83.

[12] DeOrio JK, Cofield RH (1984) Results of a second attempt at surgical repair of a failed initial rotatorcuff repair. J Bone Joint Surg Am 66(4):563-567.

[13] Watson S, Allen B, Robbins C, Bedi A, Gagnier JJ, Miller B (2018) Does the Rotator Cuff Tear Pattern Influence Clinical Outcomes After Surgical Repair? Orthop J Sports Med. 6(3):2325967118763107.

[14] Constant CR, Murley AH (1987) A clinical method of functional assessment of the shoulder. Clin Orthop Relat Res. 214:160-164.

[15] Vrotsou K, Ávila M, Machón M, Mateo-Abad M, Pardo Y, Garin O, et al. (2018) ConstantMurley Score: systematic review and standardized evaluation in different shoulder pathologies. Qual Life Res. 27(9):2217-2226.

[16] Oretorp N, Elmersson S (1986) Arthroscopy and irrigation control. Arthroscopy. 2(1):46-50.

[17] Hsiao MS, Kusnezov N, Sieg RN, Owens BD, Herzog JP (2016) Use of an Irrigation Pump System in Arthroscopic Procedures. Orthopedics. 39(3):e474-e478.

[18] Avery DM 3rd, Gibson BW, Carolan GF (2015) Surgeon-rated visualization in shoulder arthroscopy: a randomized blinded controlled trial comparing irrigation fluid with and without epinephrine. Arthroscopy. 31(1):12-18.

[19] Bomberg BC, Hurley PE, Clark CA, McLaughlin CS (1992) Complications associated with the use of an infusion pump during knee arthroscopy. Arthroscopy. $8(2): 224-28$. 
[20] Dolk T, Augustini BG (1989) Three irrigation systems for motorized arthroscopic surgery: a comparative experimental and clinical study. Arthroscopy. 5(4):307-314.
[21] Colvin AC, Egorova N, Harrison AK, Moskowitz A, Flatow EL (2012) National trends in rotator cuff repair. J Bone Joint Surg Am. 94(3):227-233.

Citation: Assoc. Prof. Olcay Güler, MD, Gürkan Gümüsssuyu, MD. Comparative Evaluation of Manual and Automated Irrigation Systems in the Arthroscopic Repair of Rotator Cuff Tears. 2021; 6(1):1-6. DOI:https://doi.org/10.20431/2456-0588.0601001.

Copyright: () 2021 Authors. This is an open-access article distributed under the terms of the Creative Commons Attribution License, which permits unrestricted use, distribution, and reproduction in any medium, provided the original author and source are credited. 